\title{
Insulin-Attenuated Inflammatory Response of Dendritic Cells in Diabetes by Regulating RAGE-PKC $\beta$ 1-IRS1-NF- $\kappa$ B Signal Pathway: A Study on the Anti-Inflammatory Mechanism of Insulin in Diabetes
}

\author{
Liding Zhao, ${ }^{1,2}$ Ya Li, ${ }^{1,2}$ Qingbo Lv, ${ }^{1,2}$ Min Wang, ${ }^{1,2}$ Yi Luan, ${ }^{1,2}$ Jiale Song, ${ }^{1,2}$ \\ Guosheng Fu $\mathbb{D}^{1,2}$ Junbo Ge, ${ }^{3,4}$ Yunzeng Zou, ${ }^{3,4}$ and Wenbin Zhang $\mathbb{D}^{1,2}$ \\ ${ }^{1}$ Department of Cardiovascular Diseases, Sir Run Run Shaw Hospital, College of Medicine, Zhejiang University, China \\ ${ }^{2}$ Key Laboratory of Cardiovascular Intervention and Regenerative Medicine of Zhejiang Province, China \\ ${ }^{3}$ Shanghai Institute of Cardiovascular Diseases of Zhongshan Hospital, Fudan University, Shanghai, China \\ ${ }^{4}$ Institute of Biomedical Science, Fudan University, Shanghai, China \\ Correspondence should be addressed to Wenbin Zhang; 3313011@zju.edu.cn
}

Received 15 November 2019; Revised 22 January 2020; Accepted 20 February 2020; Published 30 March 2020

Academic Editor: Ilaria Campesi

Copyright ( 2020 Liding Zhao et al. This is an open access article distributed under the Creative Commons Attribution License, which permits unrestricted use, distribution, and reproduction in any medium, provided the original work is properly cited.

\begin{abstract}
Background. Diabetes is associated with chronic inflammation, and dendritic cells (DCs) have proinflammatory effect in diabetes. The anti-inflammatory effect of insulin on diabetes is not entirely clear. The study aims to examine insulin-induced effects on the inflammatory response in DCs. Methods. Twenty-one C57BL/6 mice were divided into 3 groups. Streptozotocin was injected into the diabetic mice model. The bone marrow-derived DCs (BMDCs) were obtained from C57BL/6 mice. CD83, CD86, and type II major histocompatibility complex (MHC-II) of BMDCs were measured by flow cytometry. The fluctuations in the RNA levels of cytokines and chemokines were analyzed by quantitative RT-PCR. The concentrations of IFN- $\gamma$ and TNF- $\alpha$ were calculated using ELISA kits, and the proteins were detected using western blot. Results. In CD11c ${ }^{+}$DCs derived from the spleens with hyperglycemia, the expression of CD83 and CD86 in diabetic mice was significantly upregulated, coupled with a higher secretion level of cytokines and chemokines, and increased phosphorylation of NF- $\kappa \mathrm{B}$ and I $\kappa \mathrm{B}$. Insulin therapy was found to have a reversal effect on the inflammatory response and immune maturation in DCs. In AGEs-BSA-stimulated BMDCs, insulin repressed the immune maturation and downregulated the expression of RAGE, phospho-PKC $\beta 1$, and serine phospho-IRS1 in an adose-dependent manner. Such effects can be abolished by PMA, but not IR-neutralizing antibody. AGEs-BSA-induced BMDCs immune maturation was inhibited by the neutralizing antibody of RAGE, the PKC $\beta 1$ inhibitor, or the IRS1 siRNA. Conclusions. Insulin has the capability of attenuating the inflammatory response of DCs in diabetes, partly through the downregulation of RAGE expression followed by the inhibition of PKC $\beta 1$ phosphorylation and IRS1 serine phosphorylation, resulting in the inactivation of IR binding-independent NF- $\kappa$ B. This might partly explain the antiatherogenic effect of insulin on diabetes.
\end{abstract}

\section{Introduction}

The prevalence of diabetes has been increasing especially in Asian countries. The disease is associated with severe atherosclerosis $[1,2]$ and other cardiovascular diseases [3], accounting for a high rate in mortality and mobility. Low-level cellular inflammatory events $[4,5]$, such as increased tumor necrosis factor- (TNF-) $\alpha$ [6], C-reactive protein (CRP) [7], and interleukin- (IL-) 6 [6], have been reported to be associated with diabetes and considered to be mainly attributed to the onset of vascular complications.

Dendritic cells (DCs) are the only specialized antigenpresenting cells during inflammatory response [8]. Sufficient data $[9,10]$ have suggested an active role of DCs in the 
progression of atherosclerosis. In agreement with this, our previous study demonstrated a significantly higher amount of circulating active DCs in type 2 diabetic than in those nondiabetic patients with unstable angina pectoris (UAP) [11]. This observation could be due to the immune maturation of DCs induced by advanced glycosylation end-bovine serum albumin (AGEs-BSA) [12] or hyperinsulinemia [13], which in turn stimulate $\mathrm{T}$ cell proliferation and cytokine secretions. Thus, DCs may play a role in promoting inflammation and atherogenesis in diabetes.

Insulin therapy has been identified as the most effective approach in controlling hyperglycemia in diabetic patients. It was reported that insulin could exert an antiinflammatory effect $[14,15]$ by inhibiting nuclear factor(NF-) $\kappa \mathrm{B}[16]$ signaling pathway, causing antiatherogenis [17]. However, insulin could impair insulin signaling pathway, augment proinflammatory factor and oxidative stress [18], and promote macrophage foam cell formation [19], which may collectively cause the acceleration of atherosclerosis process $[20,21]$. Nevertheless, the effect of insulin therapy in attenuating the inflammatory response of DCs in diabetes is relatively unknown.

This study aims to investigate the role of insulin in attenuating the DC-mediated immune inflammatory response in diabetes and to dissect the underlying molecular mechanism.

\section{Methods}

2.1. Animals and Spleen CD11 $c^{+}$DC Separation. Twenty-one 8 -week-old C57BL/6 mice of approximately $20 \mathrm{~g}$ in weight were housed in a temperature-controlled room under a 12 hour light and dark circadian cycle, with access to water and food ad libitum (Animal Administration Center of Fudan University, Shanghai, China). Fourteen mice received an intraperitoneal injection of streptozotocin (STZ, $80 \mathrm{mg} / \mathrm{kg}$, Sigma, St Louis, MO, USA) daily for one week, after which they were maintained under the same condition for one week before their blood glucose levels were monitored. Those with fasting blood glucose levels over $13 \mathrm{mM}$ were deemed diabetic [22], seven of which received a corresponding dose of insulin subcutaneously to bring their blood glucose levels down to normal, for a total duration of 8 weeks before the body weights were measured.

The animals were anesthetized with isoflurane (5\% for induction and $1 \%$ for maintenance) for blood collection before euthanization by $\mathrm{CO}_{2}$ inhalation. Subsequently, the spleens were crushed for the purification of CD11c ${ }^{+}$DCs using anti-CD $11 \mathrm{c}^{+}$microbeads (MiltenyiBiotec, Bergish Gladbach, Germany).

All animal experimental procedures were approved by the Animal Care and Use Committee of Fudan University and performed in strict adherence to the Guide for the Care and Use of Laboratory Animals (NIH publication no. 85-23, National Academy Press, Washington, DC, USA, revised 1996).

2.2. Glucose Tolerance Test (GTT) and Insulin Tolerance Test (ITT). The diabetic mice fasted for 6 hours and 12 hours, respectively, before ITT and GTT experiments [23]. It is deployed as the basal blood glucose levels $(0 \mathrm{~min})$ before the gavage of glucose $(1.8 \mathrm{~g} / \mathrm{kg}$ bodyweight $)$ or injected intraperitoneally with insulin $(0.8 \mathrm{U} / \mathrm{kg}$ bodyweight). Additional blood glucose concentrations were then determined at time points of 30,60, 90, and $120 \mathrm{~min}$ post gavage or injected.

2.3. Cell Culture. Bone marrow-derived DCs (BMDCs) were obtained from $\mathrm{C} 57 \mathrm{BL} / 6$ mice. Bone marrow progenitors were cultured in 1640 medium containing $10 \mathrm{ng} / \mathrm{ml}$ granulocyte-macrophage colony-stimulating factor (GM-CSF; R \& D Systems, Minneapolis, MN, USA) and $1 \mathrm{ng} / \mathrm{ml} \mathrm{IL-4}$ (R \& D Systems, Minneapolis, MN, USA). The nonadherent cells were gently washed out immediately after $24 \mathrm{hrs}$. The remaining loosely adherent clusters were cultured by changing the medium with fresh medium every other day. On day 6, the cells were stimulated by $200 \mu \mathrm{g} / \mathrm{ml}$ AGEs-BSA (BioVision, Palo Alto, CA, USA) for $48 \mathrm{hrs}$, with or without the interference of insulin.

2.4. RAGE/IR Neutralization and PKC Interference. In order to understand the role of receptor for advanced glycation end products (RAGE) in the immune maturation of BMDC and to dissect the function of insulin receptor (IR) during insulin treatment, BMDCs were pretreated with $100 \mu \mathrm{g} / \mathrm{ml}$ anti-RAGE neutralizing antibody (R \& D Systems, Minneapolis, MN, USA) or $50 \mu \mathrm{g} / \mathrm{ml}$ anti-IR neutralizing antibody (GeneTex, San Antonio, TX, USA) for $1 \mathrm{hr}$ before undergoing further treatment with AGEs-BSA and insulin for $48 \mathrm{hrs}$. The effects of protein kinase C- (PKC-) $\beta 1$ on immune maturation were examined by pretreating the BMDCs with $50 \mathrm{ng} / \mathrm{ml}$ phorbol esters (PMA, Sigma, St Louis, MO, USA) for 15 mins before stimulation with AGEs-BSA and insulin or costimulation with $4 \mu \mathrm{M}$ CGP53353 (Sigma, St Louis, MO, USA) for $48 \mathrm{hrs}$. The immune maturation was examined by flow cytometry, real-time quantitative RT-PCR, ELISA, and western blot.

2.5. Cell Transfection of siRNA-IRS1. Cells were transfected with $100 \mathrm{nM}$ of siRNA-IRS1 (Applied Biosystems, Foster City, CA, USA). BMDCs $\left(5 \times 10^{5}\right)$ were seeded on 6 -well plates. The cells were mixed with $100 \mu$ l of OptiMEM (Gibco, Grand Island, NY, USA) before being pulsed using Gene Pulser Xcell ( $400 \mathrm{~V}, 150 \mu \mathrm{F}, 100 \Omega$; Bio-Rad, Hercules, CA, USA) in a $4 \mathrm{~mm}$ cuvette (Molecular BioProducts, San Diego, CA, USA) [24]. The cells were transferred to RPMI-1640 medium before an equal amount of RPMI-1640 containing $20 \%$ FBS was added $1 \mathrm{hr}$ later.

2.6. Flow Cytometry Measurement. Harvested BMDCs $\left(1 \times 10^{6}\right)$ were washed before incubation with PE-conjugated-CD83, FITC-conjugated-CD86, and APC-conjugated-MHC-II (BD Pharmingen, San Diego, CA, USA) monoclonal antibodies for 30 minutes at $4^{\circ} \mathrm{C}$. Then, the cells were washed before analysis using a flow cytometer. The cells stained with the appropriate isotype-matched immunoglobulin were used as negative controls.

2.7. Quantitative RT-PCR. Total RNA was generally extracted using TRIzol reagent (Invitrogen, Carlsbad, CA, USA). The RNA used for quantitative RT-PCR analyses 
was extracted using SYBR RT-PCR kit (Takara, Dalian, China). The primer sequences for the genes of cytokines (IL-12a, IL-6, IL-1b, IFN- $\gamma$, and TNF- $\alpha$ ), chemokines (CCL2, CCL3, CCL4, and CXCR4), and GAPDH used in this study are listed below. The relative expression levels of the genes were normalized to those of GAPDH using the $2^{-\Delta \Delta \mathrm{Ct}}$ cycle threshold.

2.8. Quantification of Cytokine Production by ELISA. The plasma and supernatant fractions of BMDCs were harvested and stored at $-80^{\circ} \mathrm{C}$ before use. The IFN- $\gamma$, TNF- $\alpha$, IL- 6 , IL- $1 b$, and CCL2 concentrations were analyzed using ELISA kits according to the manufacturer's instruction ( $R$ \& D Systems, Minneapolis, MN, USA).

2.9. Western Blot. The concentrations of total protein extracted from harvested BMDCs were determined by BCA assays. The RAGE, phosphorylated- (phospho-) NF- $\kappa \mathrm{B}$, NF- $\kappa \mathrm{B}$, phospho-I $\kappa \mathrm{B}, \mathrm{I} \kappa \mathrm{B}$, serine phospho-insulin receptor subunit-1 (IRS1) $(1: 1000$, cell signal technology, Danvers, MA, USA), serine phospho-IRS2 (1:1000, Sigma, USA), phospho-PKC $\alpha$, phospho-PKC $\beta 1$, phospho-PKC $\beta 2$ (1:300, Santa Cruz, CA, USA), and phospho-PKC $\gamma(1: 1000$, Abcam, Cambridge, UK) were examined by western blot as described previously $[25,26]$. The optical densities were analyzed using ImagePro 5.0 (Media Cybernetics, Inc., Silver Spring, MD, USA) and normalized against $\beta$-actin or GAPDH.

2.10. Statistical Analysis. The results were presented as mean \pm SEM and analyzed using one-way ANOVA followed by Fisher's LSD test for multiple comparisons using the SPSS software package, version 16.0 (SPSS Inc., Chicago, IL, USA). A $p$ value of $<0.05$ is considered as statistically significant.

\section{Results}

3.1. Insulin Therapy Lowered the Plasma Glucose Level and Increased the Body Weight of Diabetic Mice. In addition to measuring fasting blood glucose in mice, this study also identified diabetic mice through ITT and GTT experiments. After gavage of glucose, the blood glucose levels were remarkably increased at $30 \mathrm{~min}$ in two groups. Plasma glucose levels in both groups continued to decrease within 30 to $120 \mathrm{~min}$, but it failed to return to baseline after $120 \mathrm{~min}$ in the DM group (Figure 1(a)). In addition, insulin tolerance in the DM group also decreased significantly (Figure 1(b)). Diabetes mellitus mouse models were successfully established. The diabetic mice developed a significantly elevated blood glucose level (fasting and postprandial) with a reduced body weight of $30.8 \%$ compared with those of the controls. Insulin therapy significantly lowered the blood glucose to a normal level (Figure 1(c)) and stopped the loss of body weight by $35.7 \%$ (Figure 1(d)). The concentrations of IL-6, IL-1b, IFN- $\gamma$, TNF- $\alpha$, and CCL2 in the plasma of diabetic mice were increased by $185.0 \%, 199.3 \%, 133.7 \%, 157.1 \%$, and $135.8 \%$, respectively (Figure $1(\mathrm{e})$ ).

3.2. Insulin Therapy Can Reverse the Immune Maturation Promoted by $\mathrm{CD}_{11 c^{+}}$DCs in Diabetic Mice with Hyperglycemia. In this study, spleen DC, which is the most studied lymphoid tissue DCs, was selected to evaluate the immune maturation of DCs in DM mice. In spleen-derived CD11c ${ }^{+}$DCs, although the expression of MHC-II only increased marginally, CD83 and CD86 had an enhanced expression by $139.1 \%$ and $28.3 \%$, respectively (Figure 2(a)). While the expression of IL-10 decreased and proinflammatory cytokines (IL-12a, IL-6, IL-1b, IFN- $\gamma$, and TNF- $\alpha$ ) and chemokines (CCL2, CCL3, CCL4, and CXCR4) increased significantly (Figures $2(\mathrm{~b})$ and $2(\mathrm{c})$ ), phosphorylated $\mathrm{I} \kappa \mathrm{B}$ and NF- $\kappa$ B showed an enhanced expression by $237.1 \%$ and $200.1 \%$, respectively (Figure $2(\mathrm{~d})$ ). These data demonstrated an activated immune inflammatory response in the DCs of diabetic mice with hyperglycemia. However, insulin therapy was able to ameliorate the inflammatory response and immune maturation of DCs (Figure 2).

3.3. Insulin Attenuated AGEs-BSA-Stimulated Immune Maturation of BMDCs in a Dose-Dependent Manner. DCs derived from bone marrow pluripotent hematopoietic stem cells. In this study, BMDCs stimulated by GM-CSF were selected for in vitro experiments. Under the stimulation of AGEs-BSA, the expression levels of CD83 and CD86 were significantly upregulated by $65.8 \%$ and $33.7 \%$, respectively, similar to those of inflammatory cytokines, including IL-6, IL-1b, IFN- $\gamma$, and TNF- $\alpha$ and chemokines, such as CCL2, CCL3, CCL4, and CXCR4 when compared with BSA controls, while anti-inflammatory cytokine IL-10 decreased (Figures 3(a), 3(c), and 3(d)). Meanwhile, phosphorylated $\mathrm{I} \kappa \mathrm{B}$ and NF- $\kappa \mathrm{B}$ had an increased expression by $36.0 \%$ and $121.0 \%$, respectively, under the same condition (Figures 3(e) and 3(f)). Insulin exerted an inhibitory effect on the expression of CD83 and CD86 in a dose-dependent manner. For cytokines, insulin upregulated the expression of IL-10 and attenuated the expression of AGEs-BSAinduced IL- $1 \mathrm{~b}$, IFN- $\gamma$, and TNF- $\alpha$. The altered levels of TNF- $\alpha$, IFN- $\gamma$, IL- 6 , IL-1b, and CCL2 were further verified by ELISA (Figure 3(b)). Similarly, the expression of phosphorylated I $\kappa$ B and NF- $\kappa$ B also decreased significantly in-line with the dosage of insulin used (Figures 3(e) and $3(\mathrm{f})$ ). In addition, insulin at $800 \mathrm{nM}$ exerted the highest level of inhibitory effect on the immune maturation of AGEs-BSA-stimulated BMDCs.

3.4. Insulin Dose Dependently Decreased the AGEs-BSAInduced Expression of RAGE and Phosphorylation of PKC $\beta 1$ and IRS1. High ambient glucose concentrations activate PKC by increasing the formation of diacylglycerol (DAG), the major endogenous cellular cofactor for PKC activation. When PKC is activated by intracellular hyperglycemia, it has a variety of effects on gene expression. So we explored the PKC signaling pathway. The expression of RAGE was significantly augmented by $128.1 \%$ in AGEs-BSA-stimulated BMDCs when compared against BSA controls. Furthermore, while the phosphorylation of PKC $\gamma$ remains unchanged, it was upregulated by $163.2 \%, 110.7 \%$, and $84.5 \%$, respectively, for $\mathrm{PKC} \alpha, \mathrm{PKC} \beta 1$, and $\mathrm{PKC} \beta 2$. Similarly, serine phosphorylation of IRS1 was increased under the same condition. However, insulin treatment significantly decreased the expression of RAGE, phosphorylation of $\mathrm{PKC} \beta 1$, and serine 


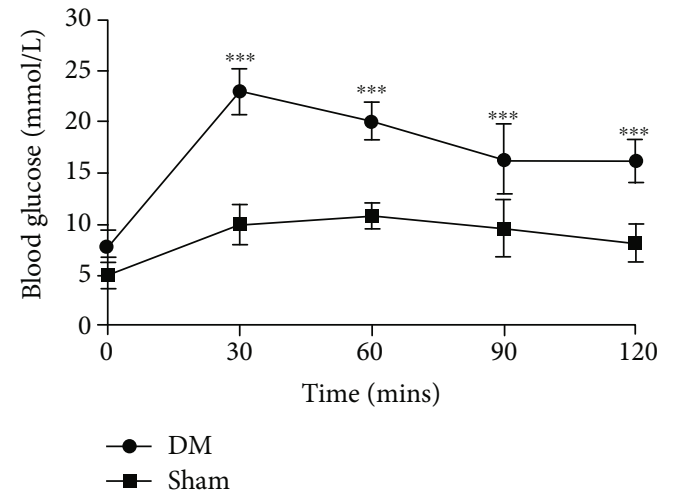

(a)

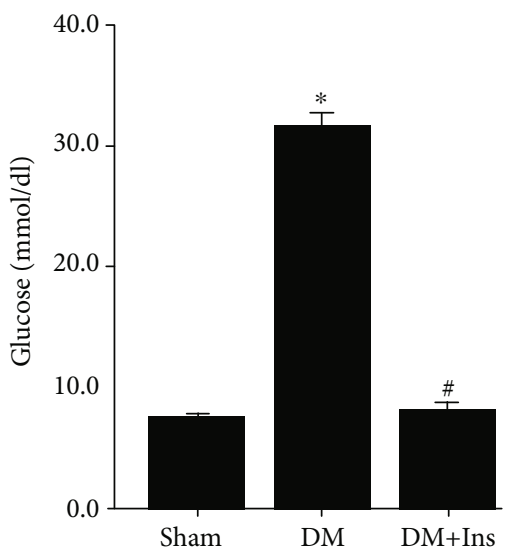

(c)

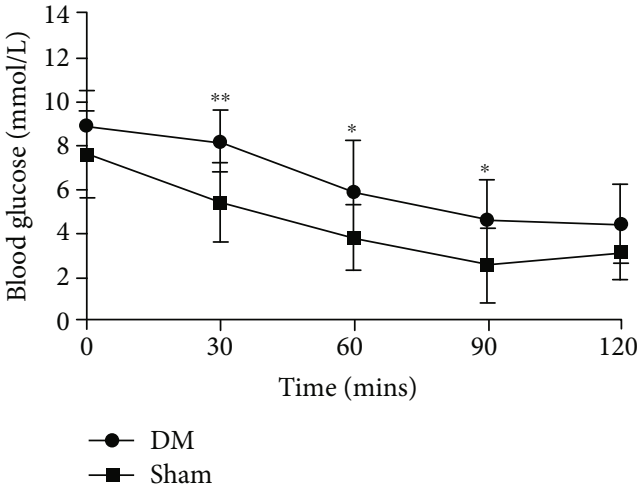

(b)
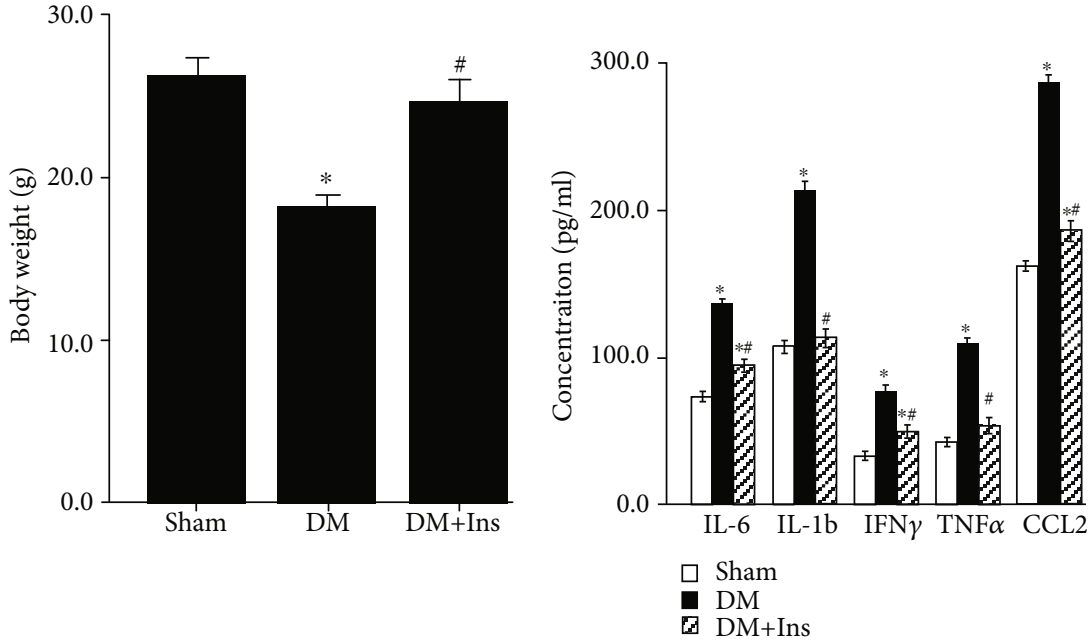

(e)

FIGURE 1: Insulin therapy improved condition in DM mice. ( $a, b)$ GTT (a) and ITT (b) experiments were performed to identify the DM mice. Glucose and insulin tolerance in the DM group decreased significantly, suggesting that diabetes mellitus mouse models were successfully established. (c-e) Compared with the Sham group, the blood glucose (c) of the DM group was significantly increased, and the weight (d) was significantly decreased. Besides, expressions of TNF- $\alpha$, IFN- $\gamma$, IL-6, IL-1b, and CCL2 in plasma analyzed by ELISA were significantly increased (e). However, insulin treatment reversed these. Data are expressed as mean \pm SED; $n=7 ;{ }^{*} p<0.05,{ }^{* *} p<0.01$, and ${ }^{* * *} p<0.001$ vs. Sham group; ${ }^{\#} p<0.05$ vs. DM group. DM: diabetes mellitus; GTT: glucose tolerance test; ITT: insulin tolerance test.

phosphorylation of IRS1 in a dose-dependent manner (Figures 3(g) and 3(h)), while the levels of upregulated PKC $\alpha$ and PKC $\beta 2$ phosphorylation remain unaltered. These observations suggested that insulin could restore the impaired signaling pathway by attenuating the stimulation effect of AGEs-BSA in the phosphorylation of PKC $\beta 1$, but not PKC $\alpha$ and PKC $\beta 2$. AGEs significantly upregulated the expression of phosphorylated IRS2, while insulin had no significantly effect on phosphorylated IRS2 (Figure 3(i)).

3.5. The Suppressing Effect of RAGE Neutralizing Antibody on AGEs-BSA-Induced Immune Maturation and Phosphorylation of PKC $\beta 1$ in BMDCs. To verify the role of RAGE in the immune maturation of AGEs-BSA-stimulated BMDCs, the expression levels of CD83, CD86, and secreted cytokines and chemotactic factors were examined with or without anti-RAGE neutralizing antibody. It was found that the upregulated CD83, CD86, and secreted cytokines and chemotactic factors were all reversed by RAGE neutralizing antibody (Figures 4(a)-4(d)). PKC activation causes activa- tion of the NF- $\kappa \mathrm{B}$ signaling pathway and increases expression of proinflammatory genes. In this study, the enhanced phosphorylation of $\mathrm{I} \kappa \mathrm{B}$ and $\mathrm{NF}-\kappa \mathrm{B}$ was decreased by $59.4 \%$ and $45.2 \%$, respectively, following treatment with antiRAGE neutralizing antibody, suggesting an active role of RAGE in AGEs-BSA-stimulated immune maturation and inflammatory response BMDCs, while a $57.3 \%$ drop of PKC $\beta 1$ phosphorylation may be attributed to RAGE. Besides, anti-RAGE neutralizing antibody also inhibits the phosphorylation of $\mathrm{I} \kappa \mathrm{B}, \mathrm{NF}-\kappa \mathrm{B}$, and $\mathrm{PKC} \beta 1$ without AGEs-BSA (Figure 4(e)).

\subsection{The Role of PKC $\beta 1$ in AGEs-BSA-Stimulated Immune} Maturation and IRS1 Serine Phosphorylation in BMDCs. Since AGEs-BSA-stimulated PKC $\beta 1$ phosphorylation in BMDCs could be attenuated by insulin, it may be one of the main underlying mechanisms during immune maturation. To verify this hypothesis, we first examined the effect of direct PKC $\beta 1$ inhibition using CGP53353 without insulin on the AGEs-BSA-stimulated immune maturation in 


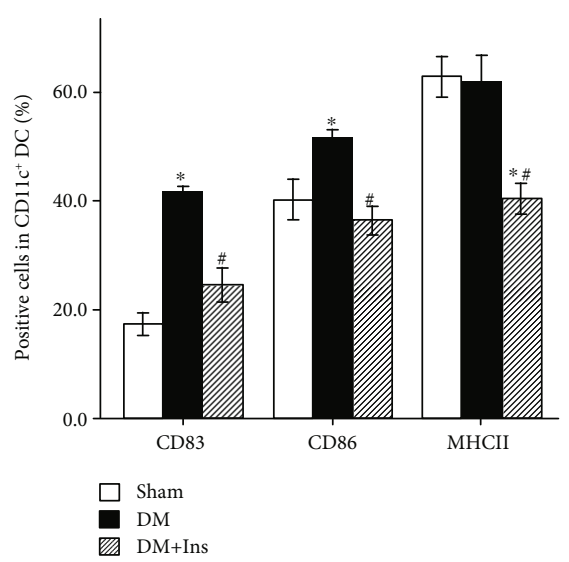

(a)

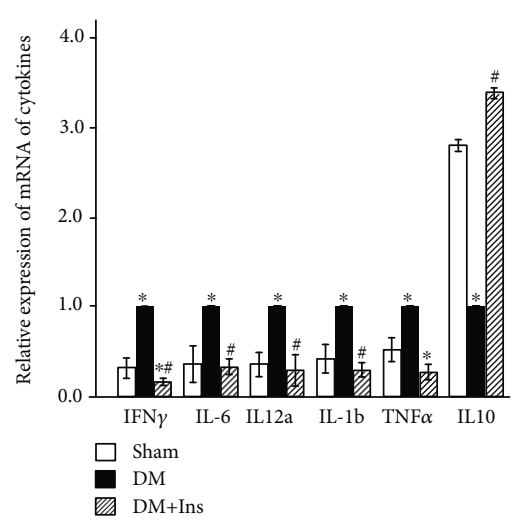

(b)

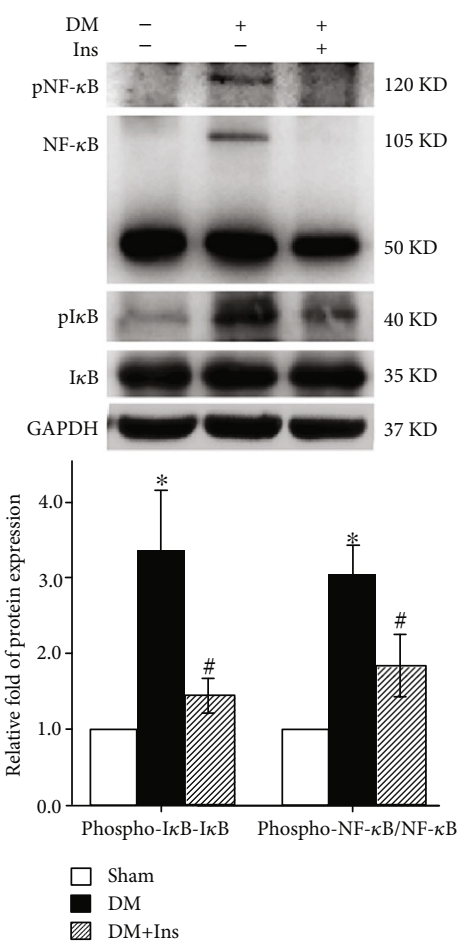

(d)

Figure 2: Insulin therapy inhibiting the immune inflammatory response of spleen DCs in DM mice. (a) Expressions of cell surface CD83, CD86, and MHC-II of spleen DCs as determined by flow cytometry. (b, c) Expressions of the cytokines and chemokines in spleen DCs were analyzed by quantitative real-time RT-PCR. Insulin treatment reduced the elevation of proinflammatory cytokines and chemokines in DM mice; insulin treatment reversed decline in anti-inflammatory factor IL-10 in DM mice. (d) Representative immunoblot analysis of $\mathrm{NF}-\kappa \mathrm{B}$ and $\mathrm{I} \kappa \mathrm{B}$ by western blot suggested the activation of NF- $\kappa \mathrm{B}$ and $\mathrm{I} \kappa \mathrm{B}$ was suppressed by insulin treatment. Data are expressed as mean \pm SED; $n=5 ;{ }^{*} p<0.05 v s$. Sham group; ${ }^{*} p<0.05$ vs. DM group. DCs: dendritic cells; DM: diabetes mellitus; TNF- $\alpha$ : tumor necrosis factor- $\alpha$; IFN- $\gamma$ : interferon- $\gamma$; MHC-II: major histocompatibility complex-II; NF- $\kappa$ B: nuclear factor- $\kappa \mathrm{B}$.

BMDCs. The results revealed that, while the upregulated expression of CD83 and CD86 was attenuated by $58.5 \%$ and $33.2 \%$, respectively, the secretion of cytokine and chemokine was also reversed in BMDCs (Figures 5(a)-5(d)). Furthermore, the phosphorylation of NF- $\kappa \mathrm{B}$ and $\mathrm{I} \kappa \mathrm{B}$ was found to be downregulated following direct PKC $\beta 1$ inhibition (Figure 5(f)). Similarly, the serine phosphorylation of IRS1 was found to be decreased upon direct PKC $\beta 1$ inhibition (Figure 5(e)). The findings suggested that PKC $\beta 1$ inhibition could repress the AGEs-BSA-stimulated immune maturation in BMDCs.

PMA, one of PKC agonists, when preconditioned for $15 \mathrm{~min}$, however, abolished the suppressing effect of insulin on AGEs-BSA-stimulated immune maturation in BMDCs, thus further verified the important role of $\mathrm{PKC} \beta 1$.

3.7. The Role of IR and IRS1 in AGEs-BSA-Stimulated Immune Maturation in BMDCs. In the insulin signaling 


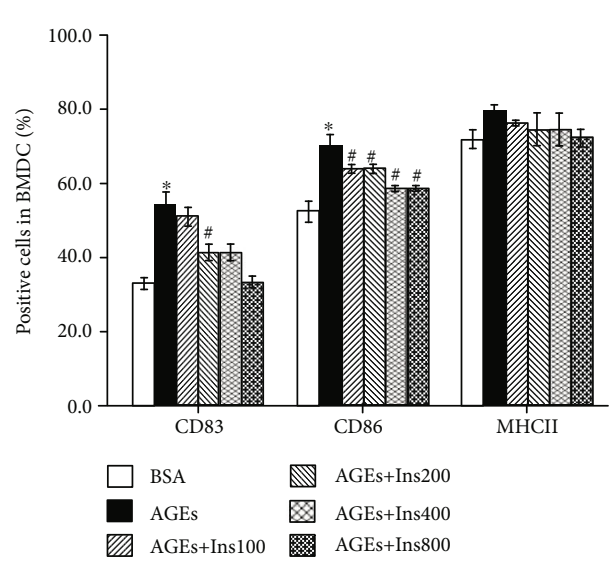

(a)

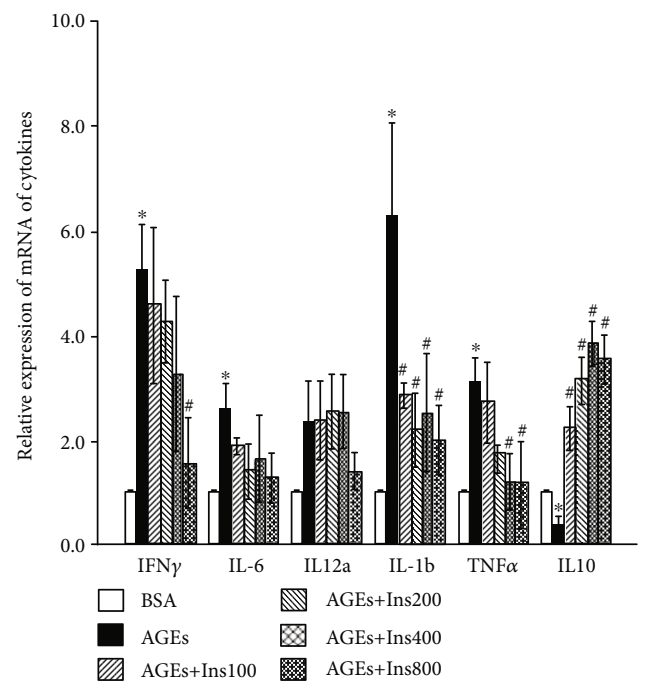

(c)

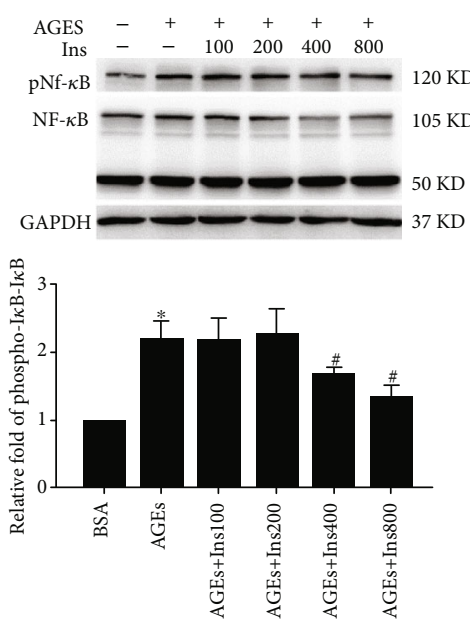

(e)

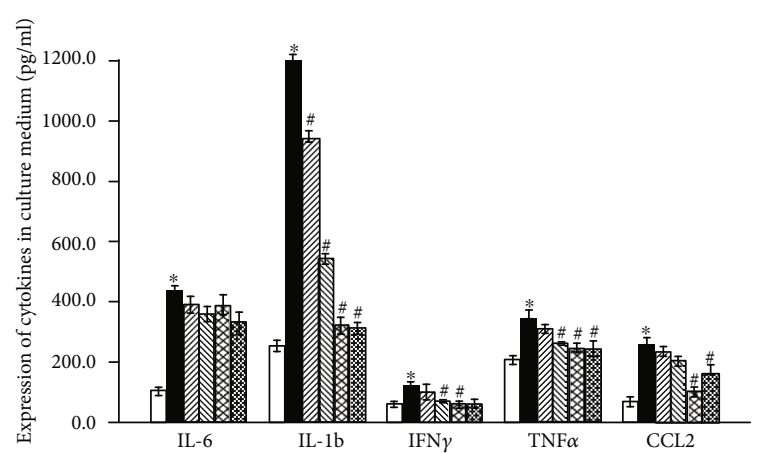

$\square$ BSA $\mathbb{N}$ AGEs+Ins200

AGEs AGEs+Ins400

UGEs+Ins100 AGEs+Ins800

(b)

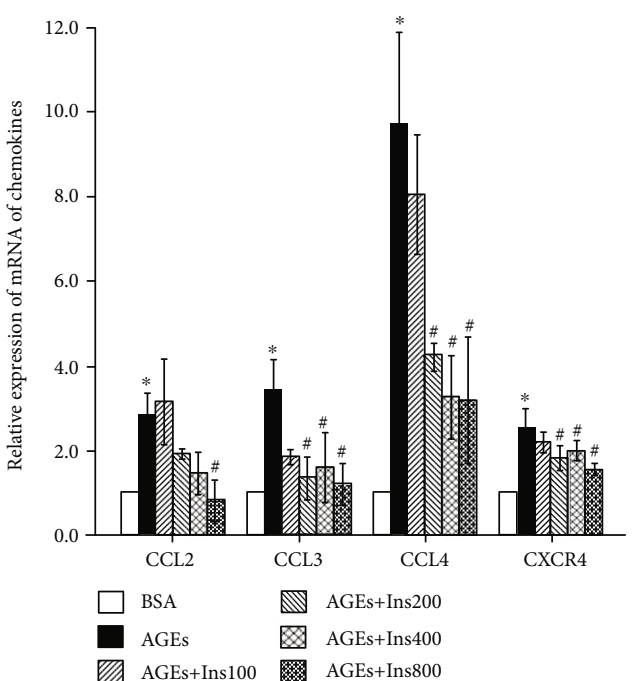

(d)

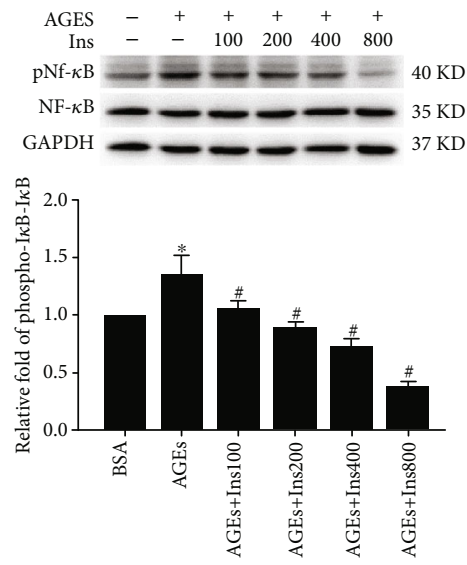

(f)

Figure 3: Continued. 


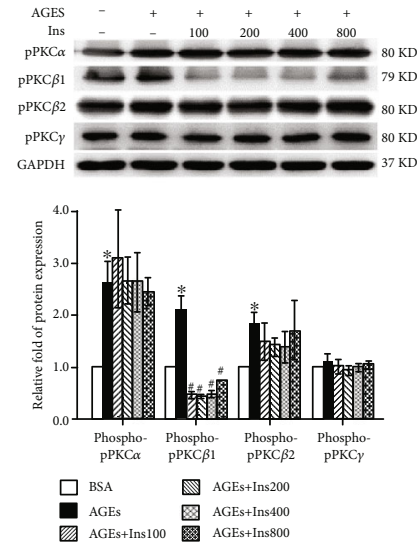

(g)

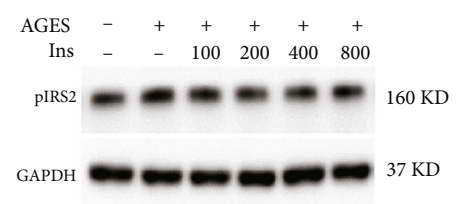

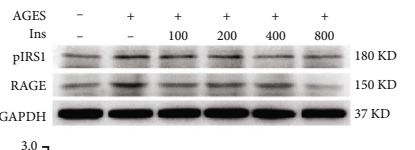

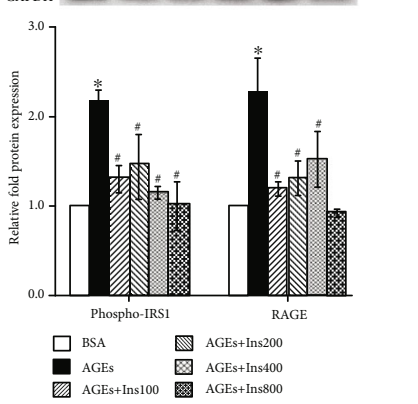

(h)

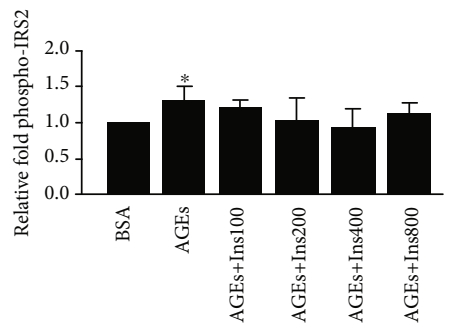

(i)

FIGURE 3: Insulin dose dependently attenuating AGEs-BSA-stimulated BMDCs immune maturation and decreasing expressions of RAGE and phosphorylation of PKC $\beta 1$ and IRS1. (a) Flow cytometric analysis was performed for cell surface CD83, CD86, and MHC-II expression examinations. Insulin exerted an inhibitory effect on the expressions of CD83 and CD86 in a dose-dependent manner. (b) The concentrations of TNF- $\alpha$, IFN- $\gamma$, IL- 6 , IL-1b, and CCL2 in the supernatants of the culture were measured by ELISA. (c, d) The expressions of cytokines and chemokines mRNA in BMDCs were analyzed by real-time quantitative RT-PCR. Results of (b-d) suggested insulin attenuated the expressions of AGEs-BSA-induced IL-1b, IL-10, IFN- $\gamma$, and TNF- $\alpha$.; (e-i) The expressions of phosphorylated and total NF- $\kappa \mathrm{B}(\mathrm{e})$, phosphorylated and total $\mathrm{I} \kappa \mathrm{B}(\mathrm{f})$, phosphorylation of PKC isoforms (g), serine phosphorylated IRS1 and RAGE (h), and serine phosphorylated IRS2 (i) were analyzed by western blot. I $\kappa$ B and NF- $\kappa$ B phosphorylation decreased significantly with the insulin treatment in a dose-dependent manner. Insulin treatment decreased significantly the upregulated expression of RAGE, phosphorylation of $\mathrm{PKC} \beta 1$, and serine phosphorylation of IRS1, not the phosphorylation of $\mathrm{PKC} \alpha$ and $\mathrm{PKC} \beta 2$. AGEs significantly upregulated the expression of serine phosphorylated IRS2, while insulin had no significantly effect on phosphorylated IRS2. Data are expressed as mean \pm SED; $n=5$; ${ }^{*} p<0.05$ vs. control group; ${ }^{\#} p<0.05$ vs. AGEs-BSA group. AGEs-BSA: advanced glycosylation end-bovine serum albumin; BMDCs: bone marrow-derived dendritic cells; RAGE: receptor for advanced glycation end products; pPKC: phosphorylated protein kinase C; IRS1: insulin receptor subunit-1; IRS2: insulin receptor subunit-2; TNF- $\alpha$ : tumor necrosis factor- $\alpha$; IFN- $\gamma$ : interferon- $\gamma$; MHC-II: major histocompatibility complex-II; NF- $\kappa$ B: nuclear factor- $\kappa$ B.

pathway, following the binding of insulin and IR, the tyrosine residue of IRS1 is phosphorylated to activate the PI3K-AKT pathway. In order to exclude the effect of IR, IR-neutralizing antibody was used with or without insulin in the AGEs-BSA-stimulated BMDCs. The results showed that the IR-neutralizing antibody had no impact on the suppressing effect of insulin (Figure 6), suggesting that the suppressing effect of insulin may be independent of traditional IR binding pathway.

In order to verify whether IRS1 plays a key role in during insulin treatment, IRS1 siRNA was applied to the AGEs-BSAstimulated BMDCs without insulin. The results revealed that, while IRS1 siRNA attenuated the expression of AGEs-BSAstimulated CD83 and CD86 by $48.3 \%$ and $30.2 \%$, respectively, the secretion of cytokine and chemokine in BMDCs was also significantly reversed (Figures 6(a)-6(d)). Furthermore, the phosphorylation of NF- $\kappa \mathrm{B}$ and $\mathrm{I} \kappa \mathrm{B}$ was found to be downregulated after IRS1 silencing (Figure 6(e)). Insulin treatment and IRS1 silencing showed no significant impact on the suppression of AGEs-BSA-stimulated immune maturation in $\mathrm{BMDCs}$ and phosphorylation of $\mathrm{NF}-\kappa \mathrm{B}$ and $\mathrm{I} \kappa \mathrm{B}$ (Figure 6(f)). Taken together, the results suggested that IRS1 silencing could repress the AGEs-BSA-stimulated immune maturation in BMDCs, thus playing a key role in the activation of NF- $\kappa \mathrm{B}$.

\section{Discussion}

Most patients with diabetes, although under treatment, tend to develop severe atherosclerosis $[1,2]$ and cardiovascular diseases [3] . Low-grade inflammation [4, 5], which is a critical event during atherosclerosis development, has been regarded as an important characteristic in diabetes. The exact mechanism of insulin therapy in antiatherogenis [17] or atherogenis [20] reported so far seem to be controversial. In addition, whether insulin therapy is able to attenuate 


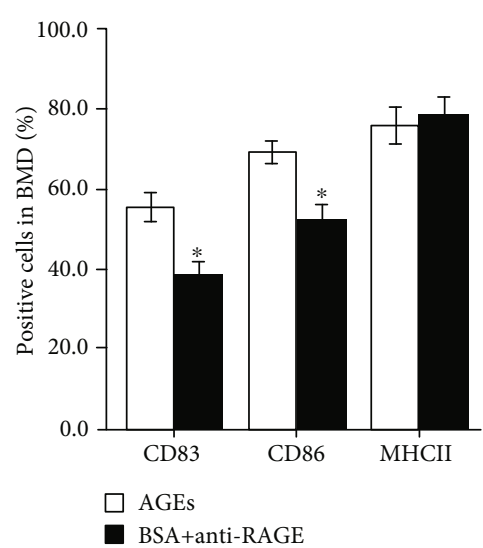

(a)

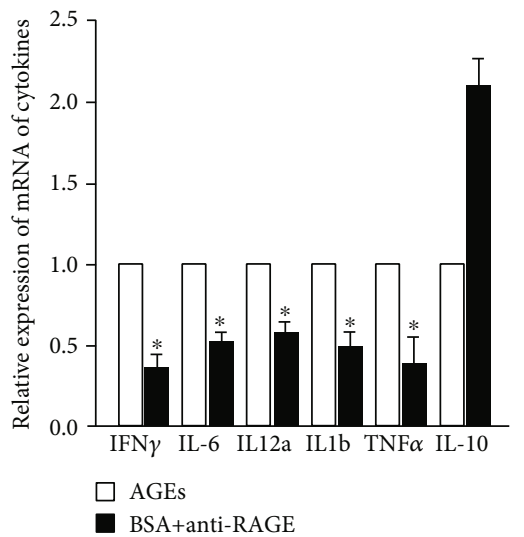

(c)

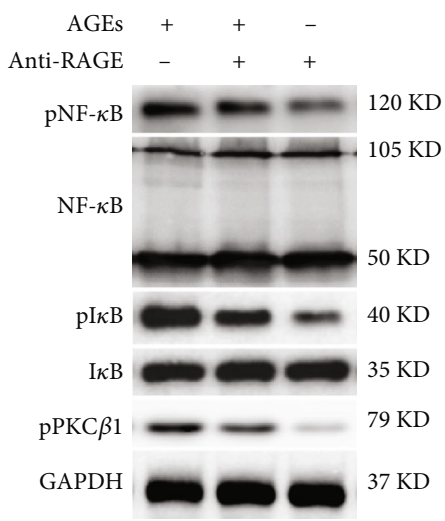

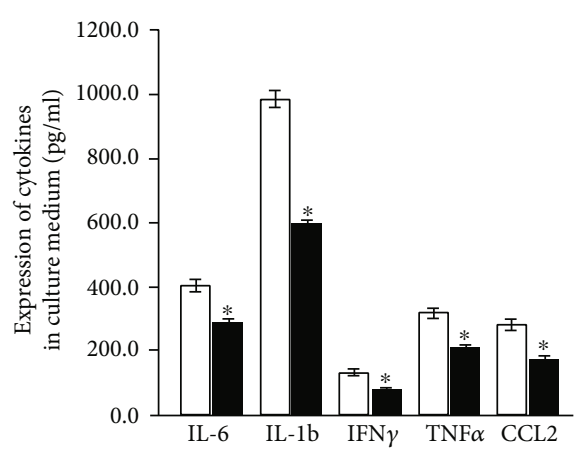

$\square$ AGEs

- BSA+anti-RAGE

(b)

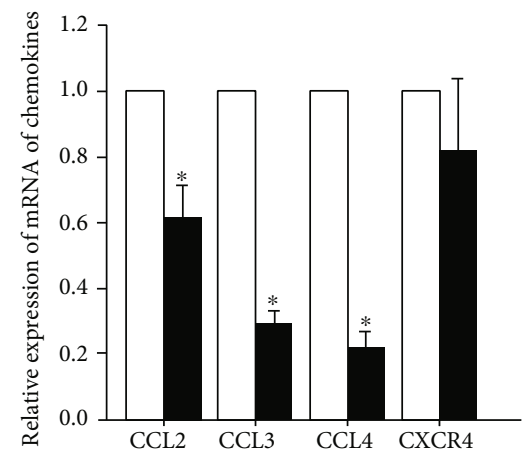

$\square$ AGEs

- BSA+anti-RAGE

(d)

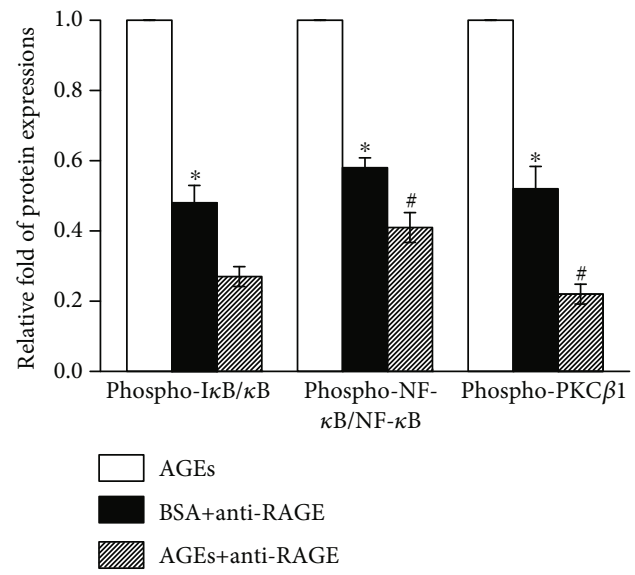

(e)

Figure 4: Role of RAGE in the AGEs-BSA-stimulated BMDCs immune maturation. (a) Flow cytometric analysis was performed for cell surface CD83, CD86, and MHC-II expression examinations. RAGE neutralizing antibody attenuated AGEs-BSA-induced upregulation of CD83 and CD86. (b) The concentrations of TNF- $\alpha$, IFN- $\gamma$, IL-6, IL-1b, and CCL2 in the supernatants of the culture were measured by ELISA; (c, d) The expressions of cytokines and chemokines mRNA in BMDCs were analyzed by real-time quantitative RT-PCR. Secretion of cytokines and chemotactic factors was all reversed by RAGE neutralizing antibody; (e) The expressions of NF- $\kappa \mathrm{B}, \mathrm{I} \kappa \mathrm{B}$, and phosphorylated PKC $\beta 1$ were determined by western blot in the presence or absence of anti-RAGE neutralizing antibody. The phosphorylation of $\mathrm{I} \kappa \mathrm{B}$, NF- $\kappa \mathrm{B}$, and PKC $\beta 1$ induced by AGEs-BSA decreased after treated with anti-RAGE neutralizing antibody. Besides, anti-RAGE neutralizing antibody also inhibit the phosphorylation of $\mathrm{I} \kappa \mathrm{B}, \mathrm{NF}-\kappa \mathrm{B}$, and PKC $\beta 1$ without AGEs-BSA. Data are expressed as mean \pm SED; $n=5$; ${ }^{*} p<0.05 v s$. control group; ${ }^{\#} p<0.05$ vs. AGEs-BSA group. AGEs-BSA: advanced glycosylation end-bovine serum albumin; BMDCs: bone marrowderived dendritic cells; RAGE: receptor for advanced glycation end products; pPKC: phosphorylated protein kinase C; TNF- $\alpha$ : tumor necrosis factor- $\alpha$; IFN- $\gamma$ : interferon- $\gamma$; MHC-II: major histocompatibility complex-II; NF- $\kappa$ B: nuclear factor- $\kappa$ B. 


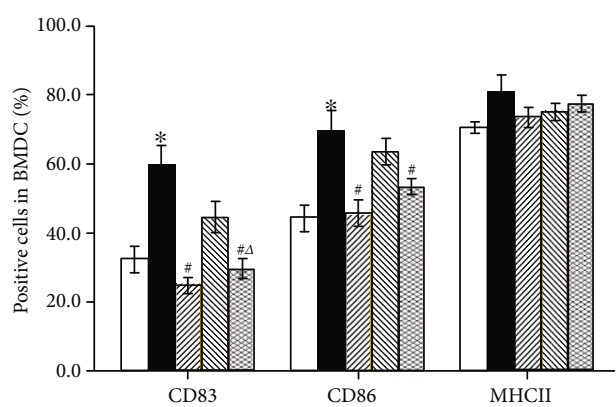

$\square{ }_{\text {AGE }} \quad \mathbb{Q}$ AGEs+Ins+

AGEs+CGP53353

(a)

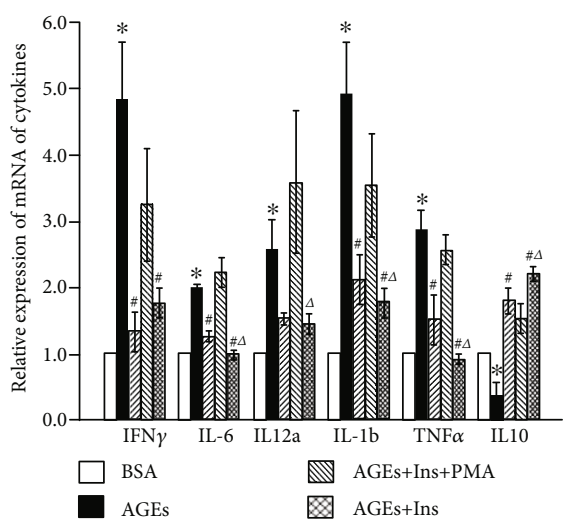

AGEs+CGP53353

(c)

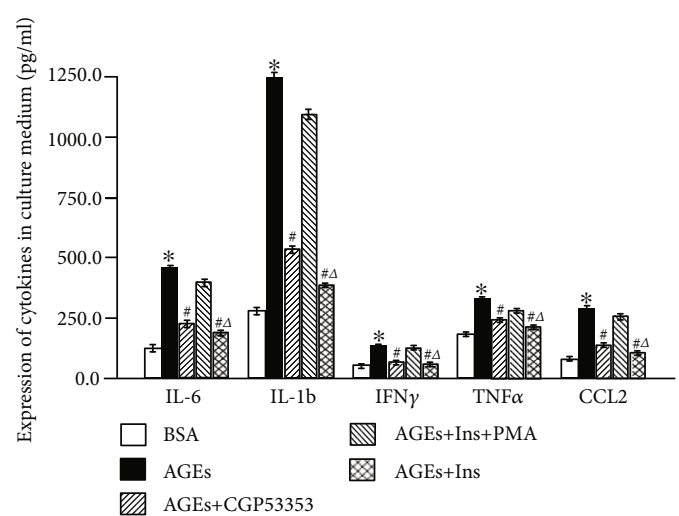

(b)

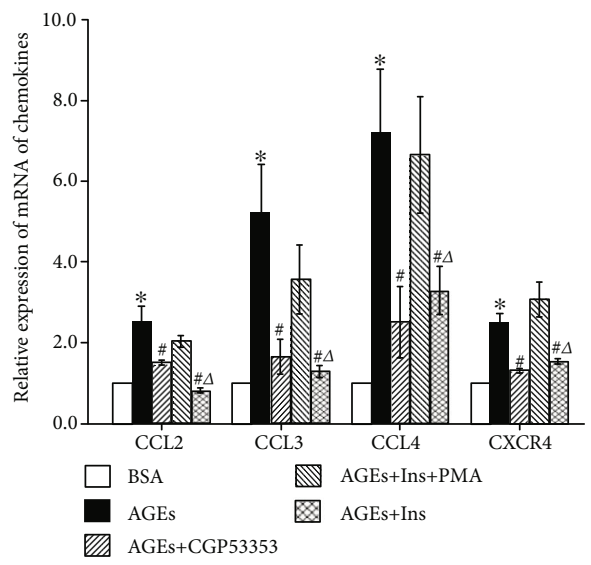

(d)

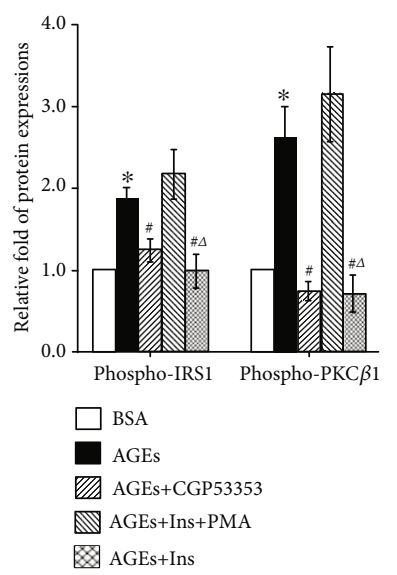

(e)

Figure 5: Continued. 


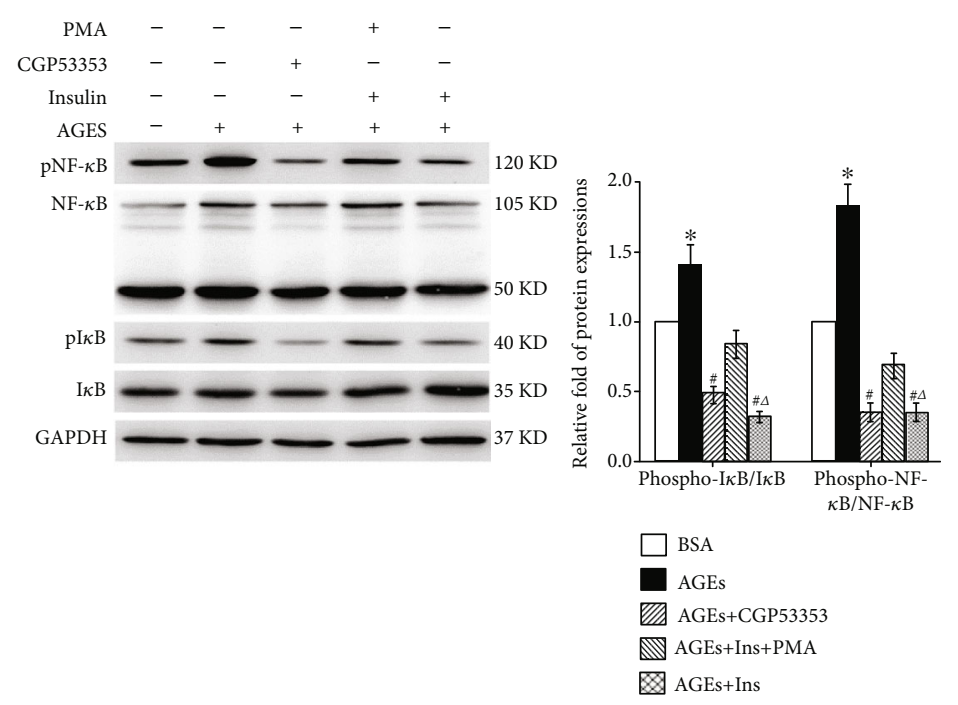

(f)

FIGURE 5: PKC $\beta 1$ played a critical role in AGEs-BSA-stimulated BMDCs immune maturation. (a) Flow cytometric analysis was performed for cell surface CD83, CD86, and MHC-II expression examinations. CGP53353 attenuated AGEs-BSA-induced CD83 and CD86 upregulation; (b) the concentrations of TNF- $\alpha$, IFN- $\gamma$, IL-6, IL-1b, and CCL2 in the supernatants of the culture were measured by ELISA; (c, d) the expressions of cytokines and chemokines mRNA in BMDCs were analyzed by real-time quantitative RT-PCR. CGP53353 attenuated proinflammatory cytokine and chemokine secretion in BMDCs and upregulated the expression of IL-10; (e, f) the expressions of NF- $\kappa \mathrm{B}$, $\mathrm{I} \kappa \mathrm{B}$, phosphorylated PKC $\beta 1$, and serine phosphorylated IRS1 were determined by western blot in the presence of PMA or CGP53353. The phosphorylation of NF- $\kappa \mathrm{B}$ and $\mathrm{I} \kappa \mathrm{B}$ were downregulated following direct PKC $\beta 1$ inhibition. In addition, the serine phosphorylation of IRS1 was found to decrease upon direct PKC $\beta 1$ inhibition. Data are expressed as mean \pm SED; $n=5 ;{ }^{*} p<0.05 v s$. control group; ${ }^{*} p<0.05$ vs. AGEs-BSA group; ${ }^{\Delta} p<0.05$ vs.AGEs + Insulin + PMA group. AGEs-BSA: advanced glycosylation end-bovine serum albumin; BMDCs: bone marrow-derived dendritic cells; RAGE: receptor for advanced glycation end products; pPKC: phosphorylated protein kinase C; IRS1: insulin receptor subunit-1; TNF- $\alpha$ : tumor necrosis factor- $\alpha$; IFN- $\gamma$ : interferon- $\gamma$; MHC-II: major histocompatibility complex-II; NF- $\kappa$ B: nuclear factor- $\kappa \mathrm{B}$.

the inflammatory response of DCs in diabetes has not been addressed.

The diabetic mice in this study developed an elevated level of plasma glucose, a reduction in body weight coupled with an increased level of plasma cytokines (TNF- $\alpha$ and IFN- $\gamma$ ), suggesting that increased inflammatory response can occur in diabetes. Moreover, the immune maturation of CD $11 \mathrm{c}^{+}$DCs from the spleens of diabetic mice was significantly upregulated, indicating that the immune maturation of DCs may play a crucial role of in diabetic inflammatory response. However, insulin therapy effectively attenuated the immune maturation of DCs, which in turn inhibited the inflammatory response in the diabetic mice. In addition, insulin can attenuate AGEs-BSA-stimulated immune maturation of DCs in a dose-dependent manner by regulating RAGE-PKC $\beta 1$-IRS1-NF- $\kappa \mathrm{B}$ signaling pathway, which is independent of IR binding. The possible underlying mechanism is illustrated in Figure 7.

Previous studies [4, 27] have shown that diabetes can develop low-level inflammation that is unrelated with obesity and poor glycemic control [4]. However, the effect of antidiabetic therapy on the attenuation of inflammation in diabetes is not entirely clear. Fantin [28] reported that $24 \mathrm{hrs}$ of continuous insulin infusion, although insignificantly, attenuated the rise of inflammation biomarkers such as IL-6 and CRP and prevented hyperglycemia in patients with diabetes who received percutaneous coronary intervention (PCI) with stent. Rakel [29] demonstrated the use of glibenclamide in controlling hyperglycemia effectively in diabetes, but there was an increased level of soluble intercellular adhesion molecule and CRP in plasma. However, sufficient data have demonstrated the use of thiazolidinedione class of oral antidiabetic agents $[30,31]$ or insulin therapy [32] in downregulating the expressions of inflammatory cytokines with success, and the effect was independent of the reduction in blood glucose as a result of the treatment [14].

The role of insulin in triggering an anti-inflammatory effect in diabetes was verified in this study. Our data demonstrated that 8-week insulin therapy could significantly downregulate plasma TNF- $\alpha$ and IFN- $\gamma$ in STZ-induced diabetic mice. Previous investigations that involved subjects of study with various diabetic time span, different antidiabetic agents, or therapeutic courses have caused much confusion in explaining the anti-inflammatory effect of antidiabetic agents, that is independent of the treatment effect in lowering the blood glucose [14]. This may also explain why intensive glycemic control failed to support macrovascular events substantially because the inflammatory mechanism was not tested in ADVANCE (Action in Diabetes and Vascular Disease: Preterax and Diamicron MR Controlled Evaluation) [33], even without increased hypoglycemia and body weight gain.

A previous literature [34] has shown an increased number of DCs in atherosclerotic arteries. AGEs were reported 


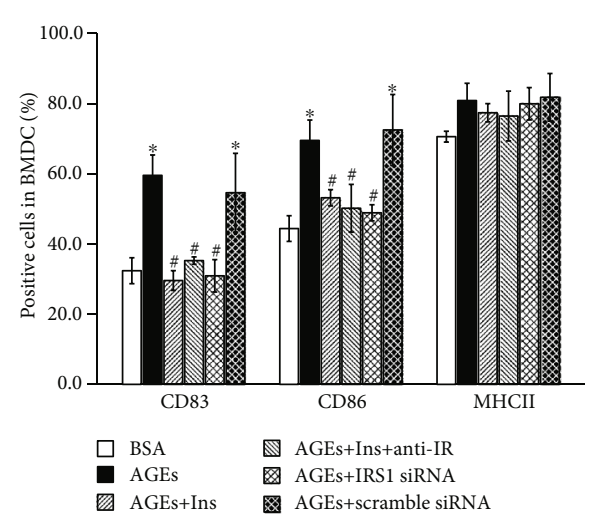

(a)

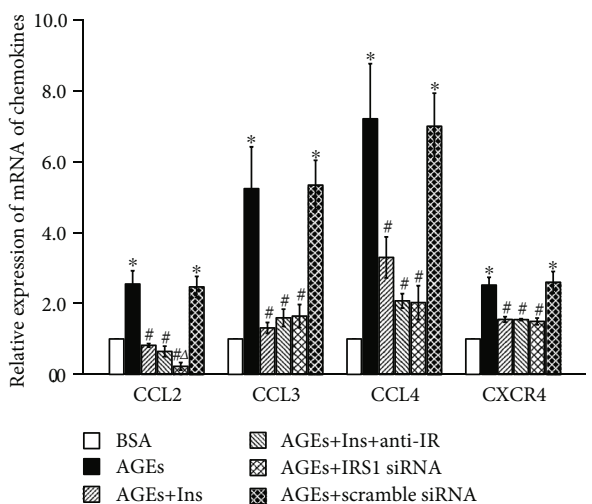

(c)

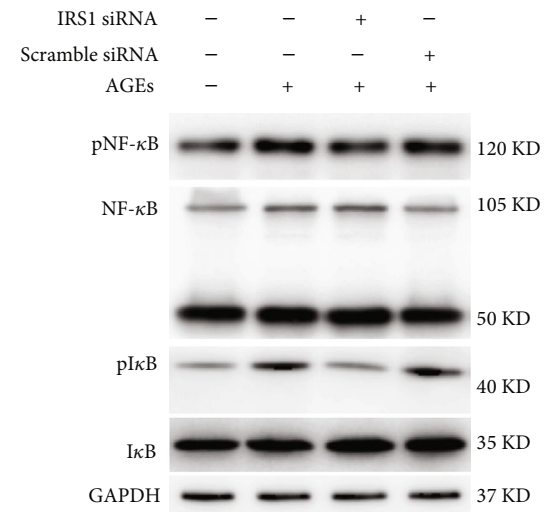

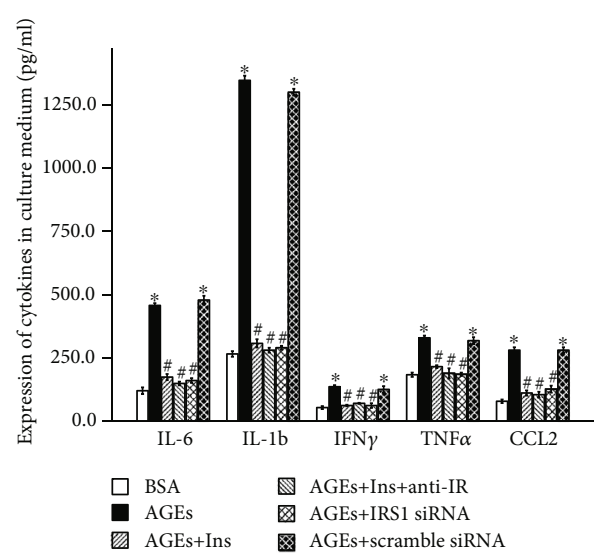

(b)

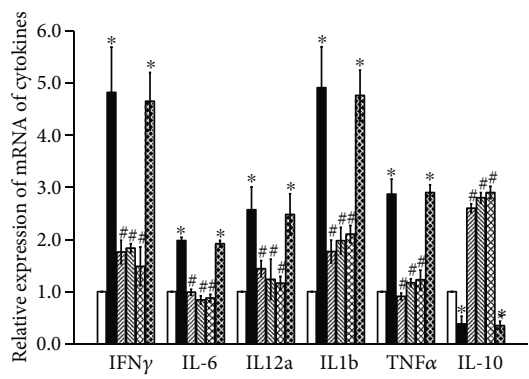

$\square$ BSA AGEs+Ins+anti-IR

aGEs AGEs+IRS1 siRnA

AGEs+Ins AGEs+scramble siRNA

(d)

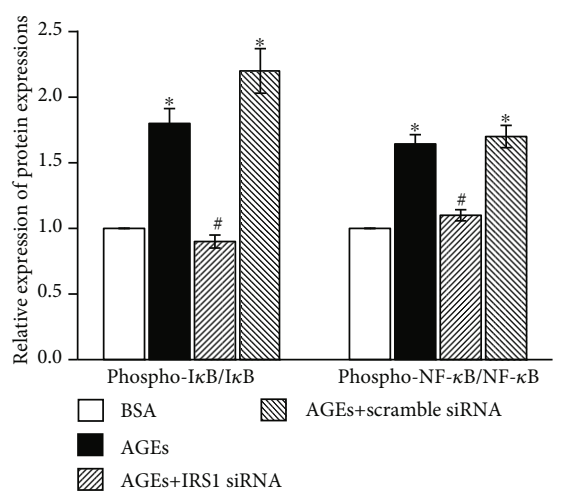

(e)

FIgURE 6: Continued. 

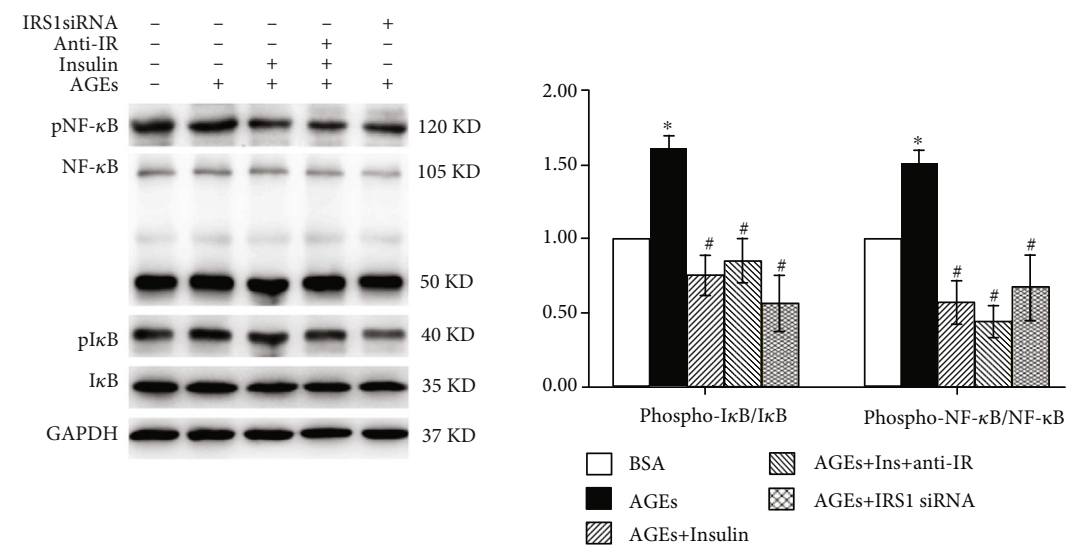

(f)

FIGURE 6: The role of IR and IRS1 in AGEs-BSA-stimulated BMDCs immune maturation. (a) Flow cytometric analysis was performed for cell surface CD83, CD86, and MHC-II expression examinations. IRS1 siRNA attenuated AGEs-BSA-induced CD83 and CD86 upregulation; (b) the concentrations of TNF- $\alpha$, IFN- $\gamma$, IL-6, IL-1b, and CCL2 in the supernatants of the culture were measured by ELISA; (c, d) the expressions of cytokines and chemokines mRNA in BMDCs were analyzed by real-time quantitative RT-PCR. IRS1 siRNA attenuated AGEs-BSA-induced the expressions of cytokines and chemokines and upregulated the expression of IL-10; (e) the expressions of NF- $\kappa \mathrm{B}$ and $I \kappa B$ were determined by western blot with the samples treated with IRS1 specific siRNA or scramble siRNA. The phosphorylation of $\mathrm{NF}-\kappa \mathrm{B}$ and I $\kappa \mathrm{B}$ was downregulated after IRS1 silencing; (f) the expressions of NF- $\kappa \mathrm{B}$ and I $\kappa \mathrm{B}$ were determined by western blot in the presence of IR neutralizing antibody or insulin or IRS1 siRNA. The results showed that the IR-neutralizing antibody had no impact on the suppressing effect of insulin. Insulin treatment and IRS1 silencing showed no significant impact on the suppression of phosphorylation of NF- $\kappa \mathrm{B}$ and $\mathrm{I} \kappa \mathrm{B}$. Data are expressed as mean $\pm \mathrm{SED} ; n=5 ;{ }^{*} p<0.05 v s$. control group; ${ }^{*} p<0.05 v s$. AGEs-BSA group; ${ }^{\Delta} p<0.05$ vs.AGEs + Insulin group. AGEs-BSA: advanced glycosylation end-bovine serum albumin; BMDCs: bone marrow-derived dendritic cells; IRS1: insulin receptor subunit-1; IR: insulin receptor; TNF- $\alpha$ : tumor necrosis factor- $\alpha$; IFN- $\gamma$ : interferon- $\gamma$; MHC-II: major histocompatibility complex-II; NF- $\kappa \mathrm{B}$ : nuclear factor- $\kappa \mathrm{B}$.

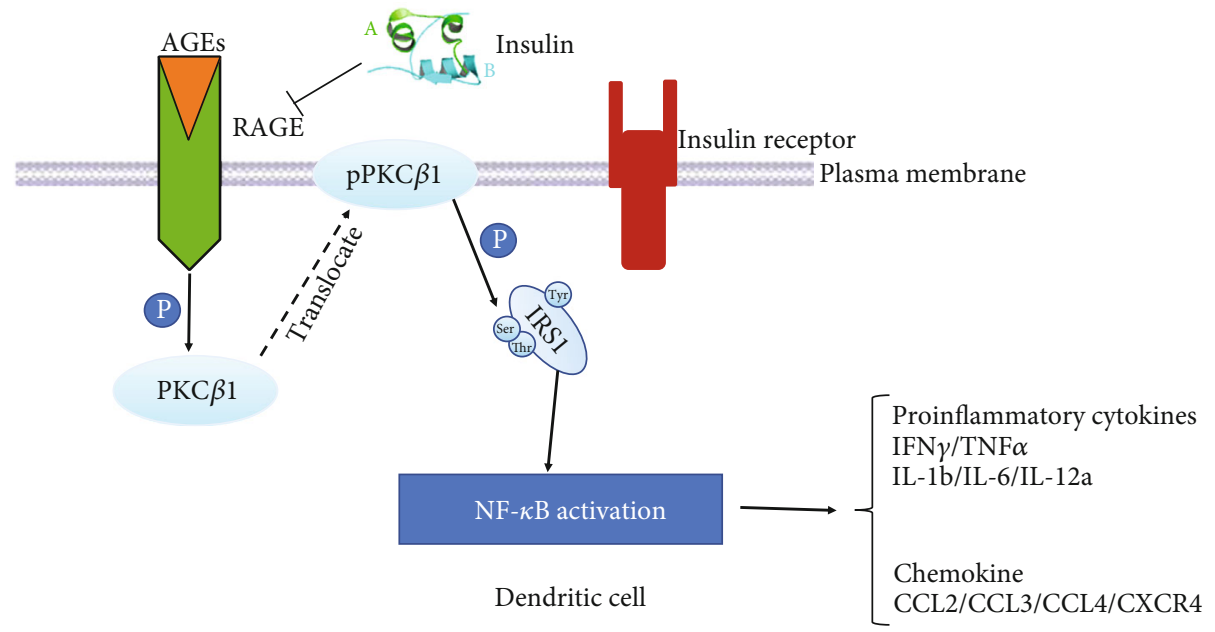

FIGURE 7: AGEs-BSA-stimulating DCs to increase RAGE expression, followed by promoted phosphorylation of PKC $\beta 1$ and serine phosphorylated IRS1 and activated NF- $\kappa$ B and upregulated proinflammatory genes; insulin therapy attenuating AGEs-BSA-induced inflammatory response of DCs partly by inhibiting RAGE expression, dephosphorylation of PKC $\beta 1$, and serine residue of IRS1.

to exert a proinflammation effect and may be associated with the onset of diabetic atherosclerosis [35]. Our previous investigation [12] has demonstrated a role of AGEs in inducing the maturation of DCs to enhance their activity in priming naive $\mathrm{T}$ cells for type 1 cytokine response and cell-mediated immunity through the upregulation of RAGE and scavenger receptor-A, partly via c-jun $n$-terminal kinase (JNK) signal- ing pathway. In this study, insulin was found to be able to dose dependently inhibit the AGEs-BSA-induced expression of CD83 and CD86; attenuate cytokines (IL-12a, IL-6, IL-1b, IFN- $\gamma$, and TNF- $\alpha$ ) and chemokines (CCL2, CCL3, CCL4, and $\mathrm{CXCR} 4)$; and decrease $\mathrm{I} \kappa \mathrm{B}$ and NF- $\kappa \mathrm{B}$ phosphorylation. Furthermore, the inhibitory effect of insulin was verified in the $\mathrm{CD} 11 \mathrm{c}^{+} \mathrm{DCs}$ from the spleen of diabetic mice treated 
with insulin. Our findings indicated that insulin therapy may exert an anti-inflammatory effect by repressing the maturation of DCs and the primary immune system, which ultimately result in antiatherogenesis.

In our previous literature [13], however, we reported that hyperinsulinemia could stimulate the immune maturation of monocyte-derived DCs via both phosphatidylinositol 3-hydroxy kinase and mitogen-activated protein kinase pathways. The discrepancies between the results from our previous findings and this study could be attributed to different antigen stimulations that are associated with different signaling pathways. In our previous study, insulin was used as the antigen to stimulate DCs by binding to the insulin receptor, which then impairs the insulin signaling pathway, to promote inflammation. In this study, AGEs-BSA was used as the antigen to stimulate DCs instead. The partial binding of insulin to the insulin receptor exerted a major inhibitory effect on the significantly upregulated expression of RAGE that was stimulated by AGEs-BSA [12], and hence neutralizing the harmful effects of inflammation. In this study, we have also identified that the suppressing effect of insulin was independent of the IR binding signaling pathway.

The findings in this study are also in agreement with the results from a previous study [12] that reported about the upregulated expression of RAGE in AGEs-BSA-stimulated DCs. The activation of protein kinase $\mathrm{C}$, which phosphorylates serine and threonine, was reported to be one of the pathophysiologic features in diabetes [36] that plays a crucial role in the development of diabetic complications [37]. Here, the upregulated PKC $\beta 1$ phosphorylation through AGEs-BSA stimulation was found to be abolished by RAGE neutralizing antibody treatment, indicating that $\mathrm{PKC} \beta 1$ phosphorylation could be regulated by RAGE. Furthermore, our results also showed that insulin treatment significantly attenuated the upregulated RAGE, causing dephosphorylation of PKC $\beta 1$. The regulation of PKC $\beta 1$ activity could contribute to the phosphorylation of serine and threonine in some key signal molecules.

With an impaired insulin signaling pathway and an increased level of inflammatory factors being the main causative factors, insulin resistance has been reported to be associated with the development of atherosclerosis [21]. It was reported [38] that glycated albumin in humans could increase the $\mathrm{PKC} \alpha$ activity and promote an increased serine/threonine phosphorylation in IRS1 and IRS2. Furthermore, PKC $\theta$ was identified to have the capacity to phosphorylate the serine residue of IRS1, and thus impair the insulin signal pathway. In this study, an increased level of serine phosphorylation in IRS1 was observed in AGEs-BSA-stimulated BMDCs, together with an increased activity of PKC $\beta 1$. However, in a similar manner to that of direct PKC $\beta 1$ inhibitor, insulin treatment significantly attenuated the serine phosphorylation in IRS1, which could then be abolished by PMA. These findings suggested that, in DCs, insulin could ameliorate the impaired insulin signaling pathway through the dephosphorylation of $\mathrm{PKC} \beta 1$, which in turn deactivates $\mathrm{NF}-\kappa \mathrm{B}$ (Figure 7 ) to exert an anti-inflammation effect. Additionally, our results also suggested $\mathrm{PKC} \beta 1$ as a potential new therapeutic target for AGEs-BSA-stimulated immune inflammation in diabetic atherosclerosis.

It is worth noting that insulin treatment significantly decreases phosphorylation of $\mathrm{PKC} \beta 1$, while $\mathrm{PKC} \alpha$ and PKC $\beta 2$ phosphorylation does not change. To the best of our knowledge, this is the first time this phenomenon has been reported. Previous studies have confirmed that insulin treatment can activate PKC $\alpha$ [39], contrary to the results of this study. In the present study, we used BMDCs for insulin treatment, not cardiomyocytes. Besides, insulin treatment time, in this study, was 48 hours instead of 30 minutes. Activation of $\mathrm{PKC} \beta$ is linked to inflammation, when $\mathrm{PKC} \beta 1$ and $\beta 2$ isoforms might be candidates for insulin receptor inhibition [40]. However, there is little research on the relationship between PKC $\beta 1 / 2$ and insulin therapy. Further research is required on these aspects.

At this stage, we are unable to demonstrate whether insulin plays a role in the downregulation of RAGE expression or interference of the binding between AGEs-BSA and RAGE. Moreover, our research was conducted based on type I diabetes mellitus, which merits further investigations by including clinical type II diabetes mellitus.

\section{Conclusion}

In conclusion, insulin therapy was proven to exert a protective effect on the inflammatory response in diabetes, partly by attenuating the immune maturation of DCs and mediating the RAGE-PKC $\beta 1$-IRS1-NF- $\kappa$ B signaling pathway. Our discovery provided additional information about the pleiotropic effect of insulin. A better understanding on the significance merits further investigation on the antiatherogenic effect of insulin in atherosclerosis in vivo.

\section{Data Availability}

The all data used to support the findings of this study are available from the corresponding author upon request.

\section{Conflicts of Interest}

The authors declare no conflicts of interests.

\section{Authors' Contributions}

W.B.Z. designed the study, contributed to the analysis, and reviewed the manuscript. L.D.Z., Ya Li, and Q.B.L. did the cell experiments, contributed to the animal experiments and analysis, and wrote the manuscript. M.W., Yi Luan, and J.S. did the animal experiments, contributed to the analysis, and edited the manuscript. All authors contributed to the discussion and reviewed or edited the manuscript. W.B.Z. is the guarantor of this work and, as such, had full access to all the data in the study and takes responsibility for the integrity of the data and the accuracy of the data analysis. Liding Zhao, Ya Li, and Qingbo Lv contributed equally to this work. 


\section{Acknowledgments}

This work was supported by the Zhejiang Natural Science Fund (LY18H020007) and the National Natural Science Foundation of China (81800212 and 81700213).

\section{References}

[1] S. I. Negi, J. S. Pankow, K. Fernstrom et al., "Racial differences in association of elevated interleukin-18 levels with type 2 diabetes: the atherosclerosis risk in communities study," Diabetes Care, vol. 35, no. 7, pp. 1513-1518, 2012.

[2] S. M. Watkins and G. S. Hotamisligil, "Promoting atherosclerosis in type 1 diabetes through the selective activation of arachidonic acid and PGE (2) production," Circulation Research, vol. 111, no. 4, pp. 394-396, 2012.

[3] M. Diamant, "Current studies of diabetic cardiomyopathy and the advancement of our knowledge: time to learn from history, guidelines, ... and other disciplines?," European Journal of Heart Failure, vol. 14, no. 2, pp. 115-117, 2012.

[4] A. Natali, E. Toschi, S. Baldeweg et al., "Clustering of insulin resistance with vascular dysfunction and low-grade inflammation in type 2 diabetes," Diabetes, vol. 55, no. 4, pp. 1133-1140, 2006.

[5] S. Ebtehaj, E. G. Gruppen, M. Parvizi, U. J. F. Tietge, and R. P. F. Dullaart, "The anti-inflammatory function of HDL is impaired in type 2 diabetes: role of hyperglycemia, paraoxonase-1 and low grade inflammation," Cardiovascular Diabetology, vol. 16, no. 1, p. 132, 2017.

[6] S. Mirza, M. Hossain, C. Mathews et al., "Type 2-diabetes is associated with elevated levels of TNF-alpha, IL-6 and adiponectin and low levels of leptin in a population of Mexican Americans: a cross-sectional study," Cytokine, vol. 57, no. 1, pp. 136-142, 2012.

[7] A. P. Kengne, G. D. Batty, M. Hamer, E. Stamatakis, and S. Czernichow, "Association of C-reactive protein with cardiovascular disease mortality according to diabetes status: pooled analyses of 25, 979 participants from four U.K. prospective cohort studies," Diabetes Care, vol. 35, no. 2, pp. 396-403, 2012.

[8] Y. J. Liu, "Dendritic cell subsets and lineages, and their functions in innate and adaptive immunity," Cell, vol. 106, no. 3, pp. 259-262, 2001.

[9] Y. V. Bobryshev, "Dendritic cells in atherosclerosis: current status of the problem and clinical relevance," European Heart Journal, vol. 26, no. 17, pp. 1700-1704, 2005.

[10] A. Zernecke, "Dendritic cells in Atherosclerosis," Arteriosclerosis, Thrombosis, and Vascular Biology, vol. 35, no. 4, pp. 763-770, 2015.

[11] K. Yao, H. Lu, R. Huang et al., "Changes of dendritic cells and fractalkine in type 2 diabetic patients with unstable angina pectoris: a preliminary report," Cardiovascular Diabetology, vol. 10, no. 1, p. 50, 2011.

[12] J. Ge, Q. Jia, C. Liang et al., “Advanced glycosylation end products might promote atherosclerosis through inducing the immune maturation of dendritic cells," Arteriosclerosis, Thrombosis, and Vascular Biology, vol. 25, no. 10, pp. 21572163, 2005.

[13] H. Lu, J. Y. Qian, K. Yao et al., "Hyperinsulinemia induced immune maturation of human monocyte derived dendritic cells: bridging between diabetes and atherosclerosis," Zhon- ghua Xin Xue Guan Bing Za Zhi=Chinese Journal of Cardiovascular Diseases, vol. 35, no. 12, pp. 1151-1154, 2007.

[14] X. M. Mao, H. Liu, X. J. Tao, G. P. Yin, Q. Li, and S. K. Wang, "Independent anti-inflammatory effect of insulin in newly diagnosed type 2 diabetes," Diabetes/Metabolism Research and Reviews, vol. 25, no. 5, pp. 435-441, 2009.

[15] Q. Sun, J. Li, and F. Gao, "New insights into insulin: the antiinflammatory effect and its clinical relevance," World Journal of Diabetes, vol. 5, no. 2, pp. 89-96, 2014.

[16] Y. C. Lee, S. Jogie-Brahim, D. Y. Lee et al., "Insulin-like growth factor-binding protein-3 (IGFBP-3) blocks the effects of asthma by negatively regulating NF-kappa B signaling through IGFBP-3R-mediated activation of caspases," The Journal of Biological Chemistry, vol. 286, no. 20, pp. 17898-17909, 2011.

[17] P. Dandona, A. Chaudhuri, H. Ghanim, and P. Mohanty, "Insulin as an anti-inflammatory and antiatherogenic modulator," Journal of the American College of Cardiology, vol. 53, 5 Suppl, pp. S14-S20, 2009.

[18] B. Cannizzo, A. Lujan, N. Estrella, C. Lembo, M. Cruzado, and C. Castro, "Insulin Resistance Promotes Early Atherosclerosis via Increased Proinflammatory Proteins and Oxidative Stress in Fructose-Fed ApoE-KO Mice," Experimental Diabetes Research, vol. 2012, Article ID 941304, 8 pages, 2012.

[19] Y. M. Park, S. R Kashyap, J. A Major, and R. L. Silverstein, "Insulin promotes macrophage foam cell formation: potential implications in diabetes-related atherosclerosis," Laboratory Investigation, vol. 92, no. 8, pp. 1171-1180, 2012.

[20] E. V. Galkina, M. Butcher, S. R. Keller et al., "Accelerated atherosclerosis inApoe-/-Mice heterozygous for the insulin receptor and the insulin receptor substrate-1," Arteriosclerosis, Thrombosis, and Vascular Biology, vol. 32, no. 2, pp. 247-256, 2012.

[21] L. Monnier, M. Hanefeld, O. Schnell, C. Colette, and D. Owens, "Insulin and atherosclerosis: how are they related?," Diabetes \& Metabolism, vol. 39, no. 2, pp. 111-117, 2013.

[22] Y. Zhang, S. A. Babcock, N. Hu, J. R. Maris, H. Wang, and J. Ren, "Mitochondrial aldehyde dehydrogenase (ALDH2) protects against streptozotocin-induced diabetic cardiomyopathy: role of GSK $3 \beta$ and mitochondrial function," BMC Medicine, vol. 10, no. 1, 2012.

[23] Y. Liu, J. Deng, and D. Fan, "Ginsenoside Rk3 ameliorates high-fat-diet/streptozocin induced type 2 diabetes mellitus in mice via the AMPK/Akt signaling pathway," Food \& Function, vol. 10, no. 5, pp. 2538-2551, 2019.

[24] C. Wu, Y. Gong, J. Yuan et al., "microRNA-181a represses ox-LDL-stimulated inflammatory response in dendritic cell by targeting c-Fos," Journal of Lipid Research, vol. 53, no. 11, pp. 2355-2363, 2012.

[25] W. B. Zhang, Q. J. du, H. Li et al., "The therapeutic effect of rosuvastatin on cardiac remodelling from hypertrophy to fibrosis during the end-stage hypertension in rats," Journal of Cellular and Molecular Medicine, vol. 16, no. 9, pp. 22272237, 2012.

[26] Z. Qiu, W. Zhang, F. Fan et al., "Rosuvastatin-attenuated heart failure in aged spontaneously hypertensive rats via PKCalpha/beta 2 signal pathway," Journal of Cellular and Molecular Medicine, vol. 16, no. 12, pp. 3052-3061, 2012.

[27] M. L. Jorge, V. N. de Oliveira, N. M. Resende et al., "The effects of aerobic, resistance, and combined exercise on metabolic control, inflammatory markers, adipocytokines, and muscle 
insulin signaling in patients with type 2 diabetes mellitus," Metabolism, vol. 60, no. 9, pp. 1244-1252, 2011.

[28] S. de Souza Fantin, M. V. Wainstein, C. A. Polanczyk et al., "Inflammatory and oxidative stress markers after intravenous insulin in percutaneous coronary intervention with stent in type 2 diabetes mellitus: a randomized controlled trial," The Journal of Clinical Endocrinology and Metabolism, vol. 96, no. 2, pp. 478-485, 2011.

[29] A. Rakel, G. Renier, A. Roussin, J. Buithieu, J. C. Mamputu, and O. Serri, "Beneficial effects of gliclazide modified release compared with glibenclamide on endothelial activation and low-grade inflammation in patients with type 2 diabetes," Diabetes, Obesity \& Metabolism, vol. 9, no. 1, pp. 127-129, 2007.

[30] R. Nesto, "C-reactive protein, its role in inflammation, type 2 diabetes and cardiovascular disease, and the effects of insulin-sensitizing treatment with thiazolidinediones," Diabetic Medicine, vol. 21, no. 8, pp. 810-817, 2004.

[31] H. Chen, R. Shi, B. Luo et al., "Macrophage peroxisome proliferator-activated receptor $\gamma$ deficiency delays skin wound healing through impairing apoptotic cell clearance in mice," Cell Death \& Disease, vol. 6, no. 1, p. e1597, 2015.

[32] C. Antoniades, D. Tousoulis, K. Marinou et al., "Effects of insulin dependence on inflammatory process, thrombotic mechanisms and endothelial function, in patients with type 2 diabetes mellitus and coronary atherosclerosis," Clinical Cardiology, vol. 30, no. 6, pp. 295-300, 2007.

[33] Action to Control Cardiovascular Risk in Diabetes Study Group, H. C. Gerstein, M. E. Miller et al., "Effects of intensive glucose lowering in type 2 diabetes," New England Journal of Medicine, vol. 358, no. 24, pp. 2545-2559, 2008.

[34] M. I. Cybulsky, C. Cheong, and C. S. Robbins, "Macrophages and dendritic cells: partners in atherogenesis," Circulation Research, vol. 118, no. 4, pp. 637-652, 2016.

[35] L. Zhang, A. Zalewski, Y. Liu et al., "Diabetes-induced oxidative stress and low-grade inflammation in porcine coronary arteries," Circulation, vol. 108, no. 4, pp. 472-478, 2003.

[36] P. Geraldes and G. L. King, "Activation of protein kinase C isoforms and its impact on diabetic complications," Circulation Research, vol. 106, no. 8, pp. 1319-1331, 2010.

[37] M. E. Sobhia, B. K. Grewal, J. Bhat, S. Rohit, and V. Punia, "Protein kinase $\mathrm{C} \beta \mathrm{II}$ in diabetic complications: survey of structural, biological and computational studies," Expert Opinion on Therapeutic Targets, vol. 16, no. 3, pp. 325-344, 2012.

[38] C. Miele, A. Riboulet, M. A. Maitan et al., "Human glycated albumin affects glucose metabolism in L6 skeletal muscle cells by impairing insulin-induced insulin receptor substrate (IRS) signaling through a protein kinase $\mathrm{C}$ alpha-mediated mechanism," The Journal of Biological Chemistry, vol. 278, no. 48, pp. 47376-47387, 2003.

[39] A. E. Hopf, C. Andresen, S. Kötter et al., "Diabetes-induced cardiomyocyte passive stiffening is caused by impaired insulin-dependent titin modification and can be modulated by neuregulin-1," Circulation Research, vol. 123, no. 3, pp. 342-355, 2018.

[40] T. Kawakami, Y. Kawakami, and J. Kitaura, "Protein kinase C beta (PKC beta): normal functions and diseases," Journal of Biochemistry, vol. 132, no. 5, pp. 677-682, 2002. 